\title{
CITIUS, ALTIUS, FORTIUS - COMMUNIS. DESPUÉS DE 127 AÑOS SE MODIFICA EL MÁS ANTIGUO DE LOS SÍMBOLOS OLÍMPICOS.
}

\author{
Carlos Eduardo Villegas-Estrada \\ Candidato a doctor Universidad de La Salle - Bogotá, Colombia \\ Miembro Comité Colombiano Pierre de Coubertin \\ carlosevillegas@hotmail.com
}

Fecha de recepción: Enero 2021

Fecha de aceptación: Mayo 2021

http://doi.org/10.15366/citius2021.14.1.001

\begin{abstract}
Resumen:
Los símbolos Olímpicos fueron aumentando su significado en la medida que los Juegos Olímpicos iban creciendo, hasta posicionarse hoy en día como parte fundamental de la filosofía Olímpica, del Movimiento Olímpico y de los mismos Juegos. El mundo globalizado contemporáneo está en constante y acelerado cambio, lo que obliga a movimientos y organizaciones que pretenden mantenerse vigentes a repensarse y resignificarse para no perder la sintonía con las pujantes nuevas generaciones de consumidores. El presente trabajo tiene como propósito reconocer el lema CITIUS ALTIUS FORTIUS, el más antiguo de los símbolos Olímpicos, en momentos en que el presidente del Comité Olímpico Internacional (COI), justamente, propone modificarlo y resignificarlo. El artículo empieza describiendo los hechos que rodean la propuesta de cambio de Thomas Bach, indaga sobre la historia del lema desde su creación, rinde tributo al creador del lema, el padre Henri Didon, subrayando la influencia que tuvo sobre el fundador del Movimiento Olímpico moderno, Pierre de Coubertin, y termina presentando una serie de ejemplos de cómo el lema ha sido utilizado desde su origen hace 127 años.
\end{abstract}

Palabras clave: citius, altius, fortius, communis, Pierre de Coubertin, Henri Didon

Title: CITIUS, ALTIUS, FORTIUS - COMMUNIS. THE OLDEST OLYMPIC SYMBOL IS MODIFIED AFTER 127 YEARS

\begin{abstract}
:
The Olympic symbols were increasing their meaning as the Olympic Games grew, until today when they have positioned as a fundamental part of the Olympic philosophy, the Olympic Movement and the Olympic Games. The contemporary globalized world is in constant and accelerated change, which forces movements and organizations that intend to remain in force to rethink and re-signify themselves so as not to lose the tune of the booming new generations of consumers. The present work aims to recognize the CITIUS ALTIUS FORTIUS motto, the oldest of the Olympic symbols, at a time when the president of the International Olympic Committee (IOC), precisely, proposes to modify and re-signify it. The article begins by describing the facts surrounding Thomas Bach's proposal for change, investigates the history of the motto since its inception, pays tribute to the creator of the motto, Father Henri Didon, underlining the influence he had on the founder of the modern Olympic Movement, Pierre de Coubertin, and ends by presenting a series of examples of how the motto has been used since its origin 127 years ago.
\end{abstract}

Key words: citius, altius, fortius, communis, Pierre de Coubertin, Henri Didon

\section{INTRODUCCIÓN}

Con seguridad cuando se publique este artículo, un nuevo lema Olímpico habrá sido aprobado en la sesión 138 del Comité Olímpico Internacional (COI), realizada en Tokio, previa a la inauguración de los Juegos Olímpicos de la trigésima segunda Olimpiada de la era moderna (siempre y cuando la COVID-19 así lo disponga). La 
historia dirá entonces, que fue el alemán Thomas Bach, actual presidente del COI, quien propuso la modificación del más antiguo de los símbolos Olímpicos; sumando así, un nuevo logro a su ya extenso legado.

El actual lema Olímpico está compuesto por las tres palabras latinas: citius, altius, fortius que significan más rápido, más alto, más fuerte. Según la Carta Olímpica (COI, 2020), a través del lema se, "expresan las aspiraciones del Movimiento Olímpico", mientras que según la página web oficial del COI, en el lema se "resume una filosofía de vida o un código de conducta que anima a los atletas a dar lo mejor de sí mismos durante la competición". De acuerdo con la interpretación del profesor Norbert Müller, en su obra maestra "Olimpismo", que recoge la selección de escritos de Coubertin, el lema olímpico tiene que ser visto, no solamente desde su sentido técnico y atlético, sino desde una perspectiva moral y educativa (2000).

La propuesta del presidente Bach fue presentada en la última sesión del COI, $\left(137^{\mathrm{a}}\right)$ en la que fue reelecto como presidente, con una abrumadora votación (93-1), para un segundo y último período de cuatro años. Justamente, en un optimista discurso de aceptación, Bach, tras invitar a los miembros a buscar metas más ambiciosas en el mundo post-coronavirus, lanzó la singular propuesta: "Aprendimos por las malas durante esta crisis del coronavirus que podemos estar a la altura de nuestro lema olímpico más rápido, más alto, más fuerte en el deporte y en la vida, sólo si trabajamos juntos en solidaridad. Por lo tanto, hoy me gustaría inspirar una discusión sobre si no deberíamos complementar este lema añadiéndole, después de un guion, la palabra juntos".

Tras la reunión del Comité Ejecutivo realizada el 21 de abril, el presidente Bach informó en rueda de prensa que su iniciativa tuvo gran acogida entre la familia Olímpica, siendo avalada por importantes organizaciones, entre las que destacó al Comité Internacional Pierre de Coubertin cuyo concepto "tuvo un significado especial para el Comité Ejecutivo". La propuesta pasará ahora al pleno de la Sesión 138 del COI para su aprobación definitiva.

Las intervenciones precisas del presidente Thomas Bach pueden ser reproducidas en el siguiente enlace: https://youtu.be/Rx9pySBlv90

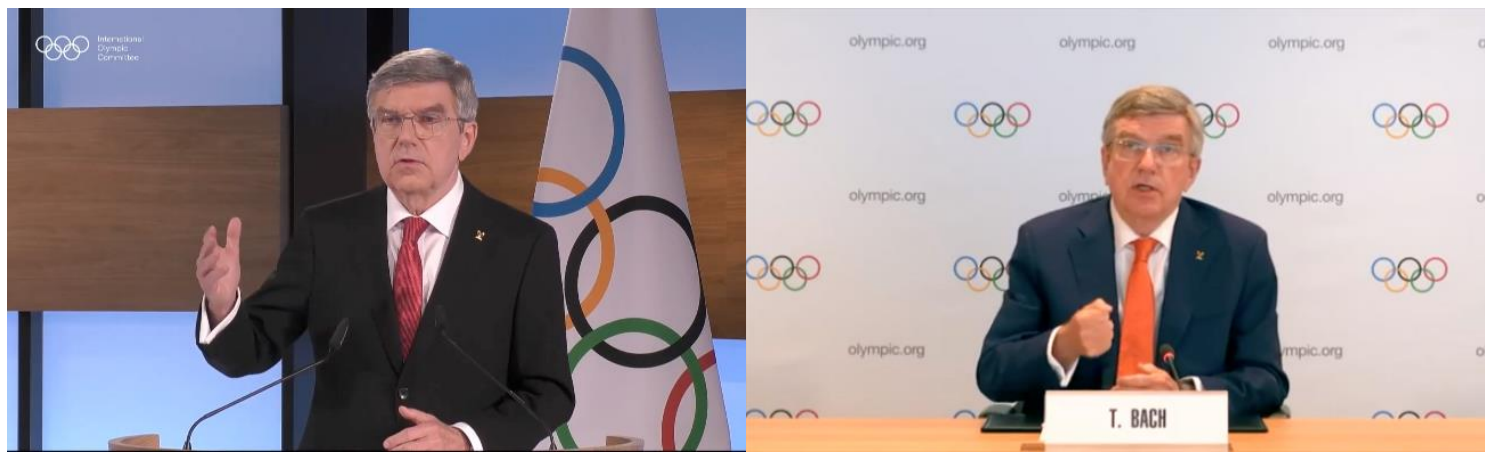

Foto \# 1 Sesión $137^{\text {a }}$ COI, marzo 11 de 2021. Foto tomada de www.olympic.com
Foto \# 2 Rueda de prensa, abril 21 de 2021. Foto tomada de www.olympic.com 


\section{HISTORIA DEL LEMA OLÍMPICO}

Pierre de Coubertin tomó prestado el lema original citius, altius, fortius de su amigo, el Padre Dominico, Henri Didon y propuso su adopción durante el congreso de París realizado en junio de 1894, en la Sorbonne Université, evento en el cual se buscaba, inicialmente, el restablecimiento de los Juegos Olímpicos y durante el cual se terminó adicionalmente fundando el Comité Olímpico Internacional.

Henri Didon y Pierre de Coubertin se conocieron el 2 de febrero de 1891 y desde aquel momento se convirtieron en coequiperos y apóstoles de una misma causa que rápidamente se fue escalando. Algunos días más tarde del primer encuentro, el 17 de marzo, Didon invitó a Coubertin a la inauguración de un festival deportivo en la Escuela Albert le Grand, en Arcueil (cerca de París) de la cual Didon era el rector. En el relato del personaje ficticio Jacques St. Clair de la novela histórica "El Idealista" de George Hirthler, se describe con precisión el discurso inaugural del festival pronunciado por Didon (Hirthler, 2016, p. 410):

\footnotetext{
"Hoy, como les he dicho a los atletas de Albert le Grand, no sólo corremos por nosotros. Nos tomamos los campo y bosques para ejercitar todos los dones que nuestro Padre celestial nos ha dado; todos los dones que podemos dominar entrenando más duro para correr más rápido, levantando nuestros ojos hacia las más altas posibilidades de los talentos que poseemos y ejercitando nuestras fortalezas y sacando aún más fuerza de nuestros equipos, individual y colectivamente. Les digo hoy, a cada uno de ustedes que está aquí para competir: pueden ir más rápido, pueden llegar más alto, pueden ser más fuertes. Citius, altius, fortius -más rápido, más alto, más fuerte- para la gloria de Dios y el honor de su escuela. Este es el lema de Albert le Grand, pero también es una llamada fresca a cada uno de ustedes para que den lo mejor de sí mientras corren hoy con sus hermanos en el deporte".
}

Coubertin que para ese entonces era el Secretario General de la Asociación Francesa de Clubes Deportivos Estudiantiles (USFSA) ofició activamente como director de la competición; de la cual tuvo que haber quedado muy impresionado y satisfecho. Coubertin "guardó ese lema en un rincón de su mente" (Kristy, 1995), a tal punto que, pocos días después, citó las palabras de Didon en un reportaje concedido a la revista especializada "Les Sport Athlétiques” (Müller \& Schwank, 2008)

De acuerdo con varias fuentes, el lema original era Citius, Fortius, Altius, aunque no se ha podido comprobar con certeza la razón por la cual se habría cambiado el orden. Una teoría sugiere que el cambio tendría que ver con la redacción en lengua latina (Müller, 2000); mientras que otros teóricos sugieren, empíricamente que, para ser más fuerte, primero hay que ir más lejos y más alto.

\section{LE PÉRE LOUIS HENRI DIDON (1840-1900)}

El monje Didon era otro visionario, pedagogo y humanista que, como de Coubertin, estaba obsesionado por incluir el deporte en el currículo académico de las escuelas francesas. Nació el 17 de marzo de 1840 en Touvet, Francia y murió pocos días antes de cumplir 60 años. Durante los últimos 10 años de su vida ejerció una gran influencia en la vida del Barón, para quien Didon resultó ser un verdadero "enviado de Dios" (Hirthler, 2016).

La conexión entre Coubertin y Didon fue inmediata, sus ideas estaban totalmente alineadas, por lo que su relación fue intensa y de mutuo apoyo. Para el escritor David Miller, Coubertin le dio a Didon una nueva perspectiva sobre el desarrollo de la sociedad, 
mientras que Didon fortaleció la determinación de Coubertin a través de su espiritualidad (Miller, 2008).

Didon fue un rebelde sacerdote que promovía la educación física y el deporte como parte del programa educativo y buscaba la integración entre escuelas privadas y públicas, ambos propósitos resistidos por los colegios religiosos de la época (Hirthler, 2016, p. 406), a tal extremo que el arzobispo lo desterró a Corsica, desde donde aprovechó el tiempo para escribir "La vida de Cristo" publicada en 1890 tras su regreso a la vida pública en París (Hirthler).

Didon fue un predicador y orador extraordinario que incorporaba una buena dosis de ironía para transmitir su poderoso mensaje, tanto desde el púlpito como frente a sus alumnos. Para Coubertin, sin embargo, su uso audaz del lenguaje fue más "encantador que perturbador", sembrando siempre "amor y verdadero liberalismo" (Müller).

En el obituario de su amigo Didon, Coubertin destacó su fortaleza moral y coherencia en la vida, expresadas a través de sus obras más que de sus palabras; "la lucha fue su mayor pasión", la cual libró inclusive desde el púlpito expresó Coubertin y agregó "para él no había retroceso, ni arrepentimiento por el pasado, ni miedo o reticencia con respecto al futuro. (Müller, 2000, p. 210).

\subsection{Didon en la escena Olímpica}

Didon se convirtió en gran promotor de la causa Olímpica, a la que contribuyó a través de su presencia en varios eventos como muestra del aprecio que tenía por Coubertin. En 1896 fue invitado de honor a la ceremonia de inauguración de los Juegos Olímpicos de Atenas. En la detallada descripción que hace Tarassouleas, en su inédito libro Olympic Games in Athens 1896-1906, a las 3 pm del lunes 25 de marzo (6 de abril) de 1896, ${ }^{1}$ el primer ministro Theodoros Dhilighiannis y el presidente del Parlamento, Alexandros Zaimis, con el Concejo de ministros entraron al estadio y ocuparon sus sillas al lado de la familia Real en el Sfendoni. Al lado derecho estaban miembros del Parlamento, del Sínodo Sagrado y "algunos religiosos extranjeros, entre quienes se encontraba el predicador Francés Peré Didon” (Tarassouleas, 1988).

En 1897 Didon fue invitado por Coubertin como orador principal al $2^{\circ}$ Congreso Olímpico realizado en Le Havre. El discurso de Didon, pronunciado durante el cuarto día del Congreso versó sobre "las cualidades del deporte [para] forman el carácter" en el que dio una explicación pedagógica sobre las actividades deportivas que Didon consideraba esenciales para los niños en la escuela (Müller, 1994).

\section{CITIUS ALTIUS FORTIUS}

Tanto el lema Olímpico como el Padre Didon se convirtieron en protagonistas del movimiento Olímpico y de los Juegos, apareciendo inscritos, entre otros, en monumentos, carteles, monedas y medallas Olímpicas y dando título a obras de arte, escuelas, cátedras y hasta publicaciones que han adoptado sus nombres.

Varias de las más emblemáticas imágenes de los escenarios deportivos de los

\footnotetext{
${ }^{1}$ En 1896 Grecia se regía aún por el calendario Juliano; a partir de 1923 adoptó al actual calendario Gregoriano, resultando en el aumento de 13 días. Así las cosas, al 15 de febrero de 1923 le siguió el 1 de marzo.
} 
Juegos Olímpicos, principalmente durante las ceremonias de inauguración, han incluido el lema Olímpico (fotos 3 y 4); por ejemplo, en el estadio Olímpico de Ámsterdam, sede de los Juegos de 1928, el lema se ha inmortalizado en su fachada, exactamente en la entrada principal donde aparece debajo de los aros olímpicos (foto 5), a pocos metros de la torre de maratón que soporta el pebetero Olímpico donde se encendió por primera vez la llama olímpica en 1928 (foto 6).

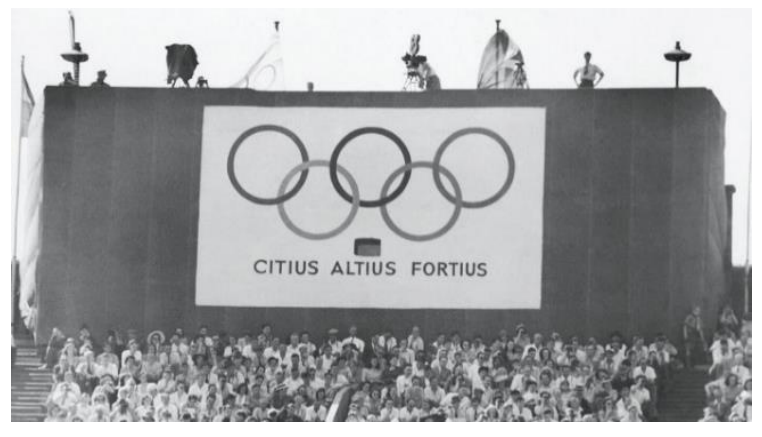

Foto \# 3 Estadio Olímpico. Recuperada de www.olympic.com

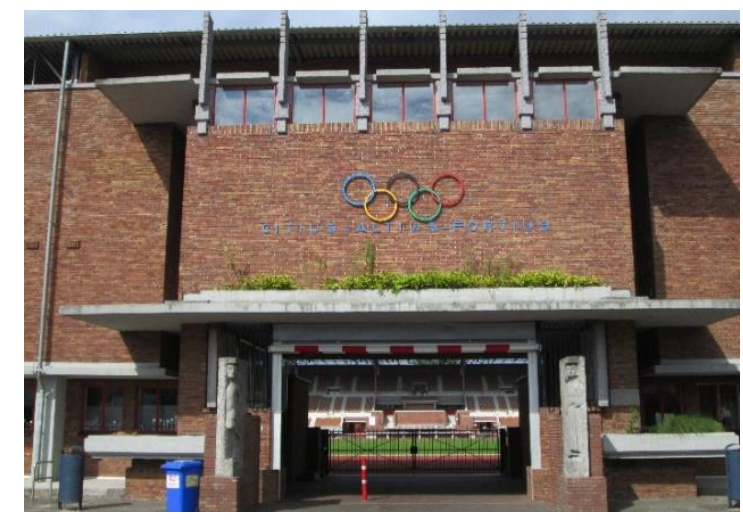

Foto \# 5 Estadio Olímpico de Ámsterdam 1928. Foto archivo personal Carlos E. Villegas
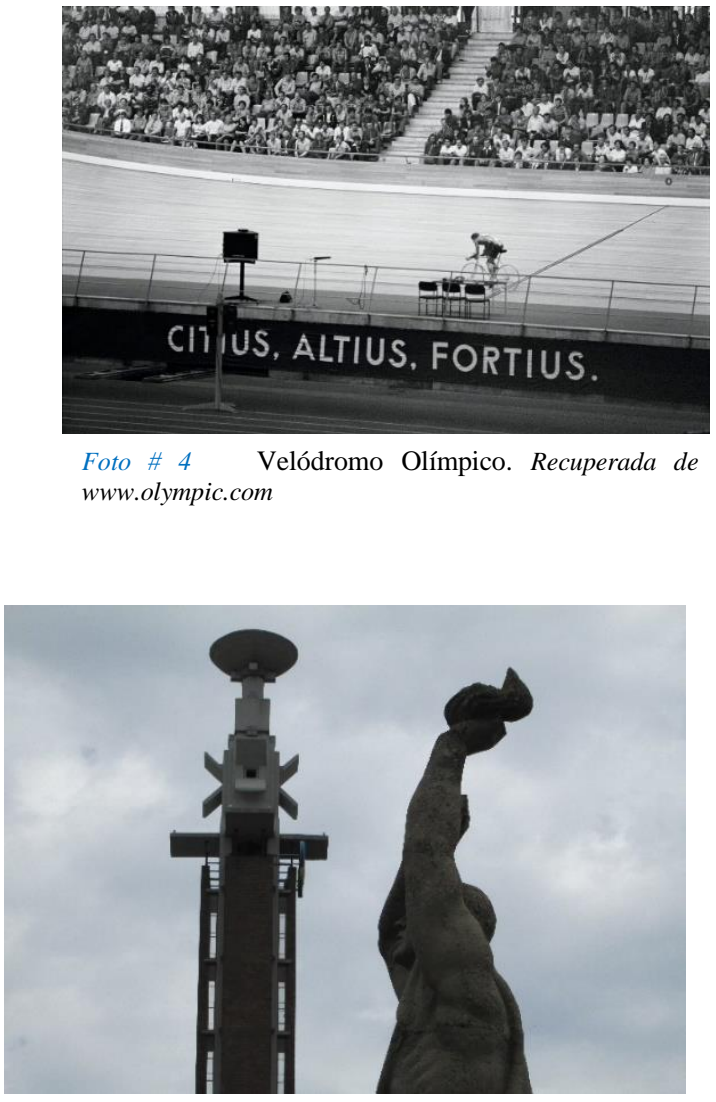

Foto \# 6 Pebetero Torre de Maratón Ámsterdam. Foto archivo personal Carlos E. Villegas

En el campo de la filatelia cuyo vínculo con el Movimiento Olímpico es tan viejo como los mismos Juegos Olímpicos es frecuente encontrar el lema olímpico: sellos postales o estampillas han sido emitidas para promover e inclusive financiar los Juegos, como sucedió en Atenas 1896. Para David Maiden, ${ }^{2}$ la filatelia es una importante fuente de referencias históricas: "las estampillas postales son story tellers, pequeñas obras de arte cuyos temas y diseños abren una ventana hacía la realidad social y política de la época en la que fueron emitidas" (Barion, 2014).

Una de las emisiones más significativas es la del aerograma emitido por el servicio postal de El Vaticano el 19 de junio de 2000 para conmemorar el centenario de la muerte de Henri Didon; la imagen del ilustrador Irio Ottavio Fantini representa al padre Didon con unos atletas en el fondo enmarcados por la inscripción CITIUS ALTIUS FORTIUS. La estampilla impresa incluye la bandera olímpica y el valor de 1.500 liras. Se imprimieron 70 copias del aerograma (Vatican-Philatelic-Society, 2000).

\footnotetext{
${ }^{2}$ David Maiden fue miembro de la antigua Comisión de Filatelia, Numismática y Memorabilia del COI.
} 
Monedas conmemorativas emitidas por las autoridades monetarias en distintos países recurren frecuentemente a los símbolos Olímpicos; en los Estados Unidos, por ejemplo, la participación de Jesse Owens en los Juegos Olímpicos de Berlín, 1936 fue perpetuada a través de una moneda conmemorativa (foto 8 ).

El lema Olímpico también está presente en el diseño de 5 de las medallas de los Juegos Olímpicos, principalmente de invierno: St. Moritz 1948 (foto \# 9), Oslo 1952, Cortina D’Ampezzo 1956 y Squaw Valley 1960; además de las de los Juegos de la Olimpiada, de Estocolmo $1956^{3}$ cuyo diseño del reverso, donde aparece el CITIUS ALTIUS - FORTIUS estuvo a cargo del artista Griego Vasos Filares (COI, medallas olímpicas) (foto 10).

Otras manifestaciones artísticas también han acudido al lema olímpico como fuente de inspiración. Una de las obras de arte que más sobresale en los jardines del Museo Olímpico en Lausana es el torso de un atleta de grandes proporciones denominada Citius, Altius, Fortius creación del artista español Miguel Ortiz Berrocal en 1992. La obra está compuesta por 6 partes movibles mecánicamente que atrae con su movimiento la atención de los visitantes al museo (Ramos Vicent, 2012).

Para finalizar con los más reconocidos usos del lema Olímpico, se destaca en el mismo Museo Olímpico, el monumento a la llama Olímpica eterna (Foto 12), el artista catalán André Ricard introdujo el lema CITIUS ALTIUS FORTIUS grabado en letras de piedra en relieve, ubicadas en la base del monumento, separadas por los aros Olímpicos (Ramos Vicent, 2012).

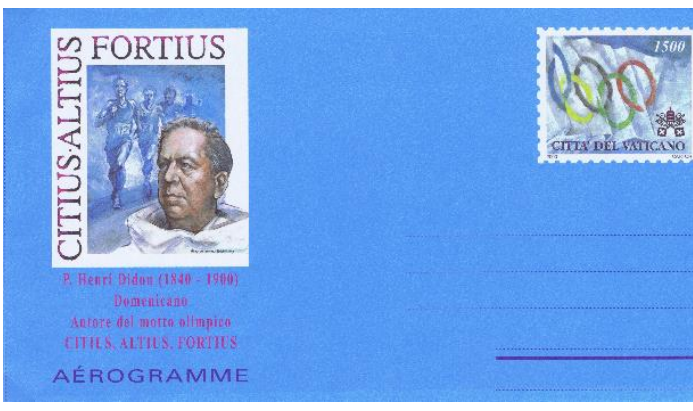

Foto \# 7 Aerograma Ciudad del Vaticano Año 2000. Foto The Journal of the Vatican Philatelic Society
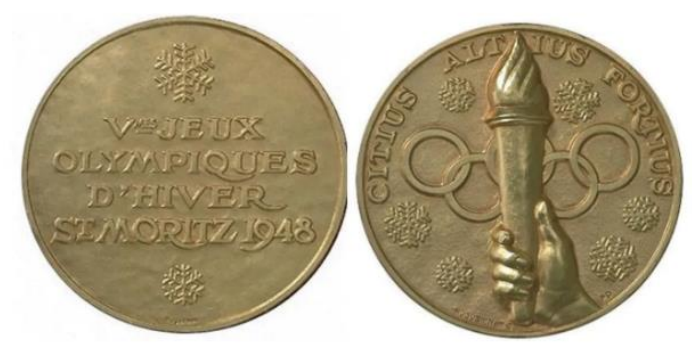

Foto \# 9 Medalla JJOO St. Moritz 1948.Foto tomada de www.olympic.com
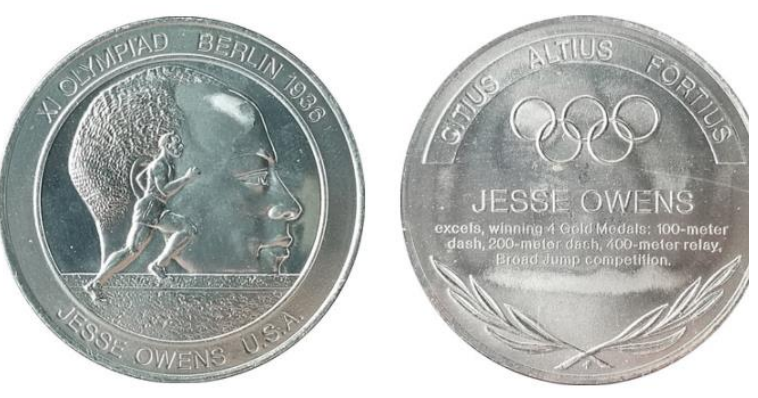

Foto \# 8 Moneda conmemorativa Jesse Owens Foto tomada de https://en.numista.com @ Jlhare
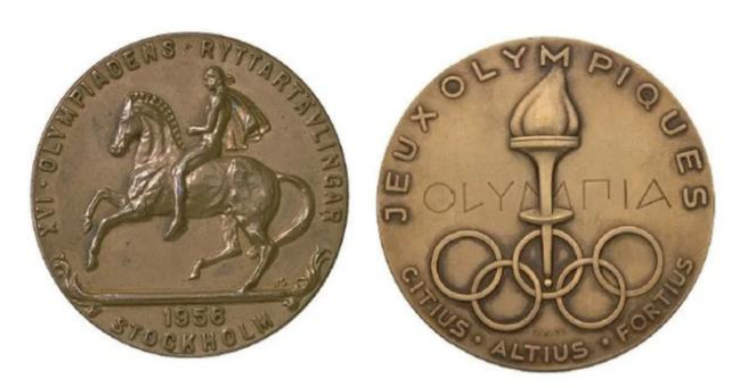

Foto \# 10 Medalla JJOO Estocolmo 1956 (Ecuestres). Foto tomada de www.olympic.com.

\footnotetext{
${ }^{3}$ Las pruebas de ecuestres de los Juegos Olímpicos de 1956 se realizaron en Estocolmo, donde diseñaron las medallas específicamente para estas pruebas.
} 


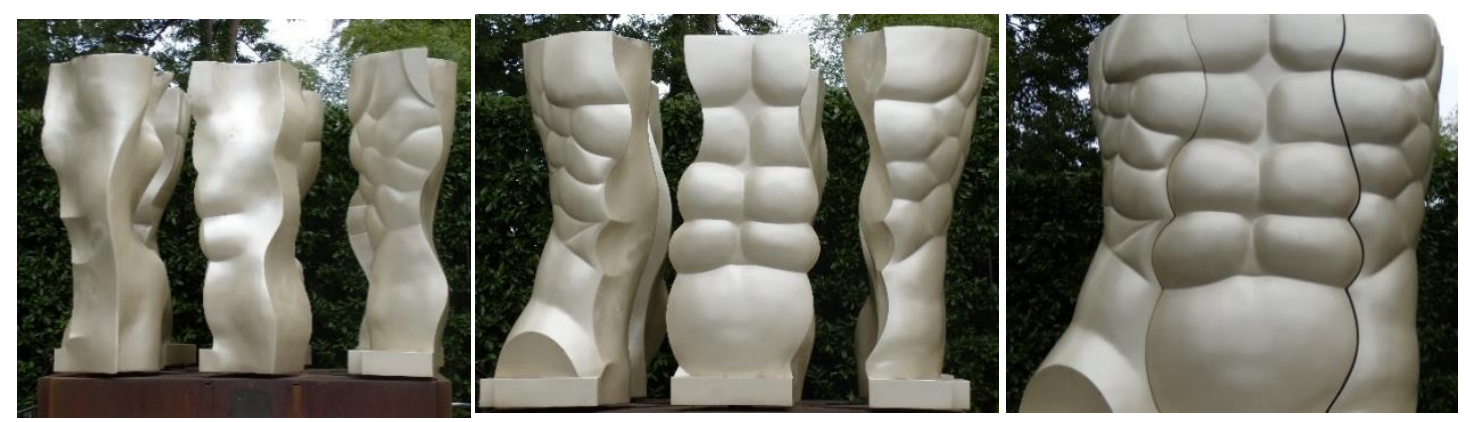

Foto \# 11 Citius Altius Fortius, Jardines del Museo Olímpico - Miguel Ortiz Berrocal, 1992. Foto archivo personal Carlos E. Villegas

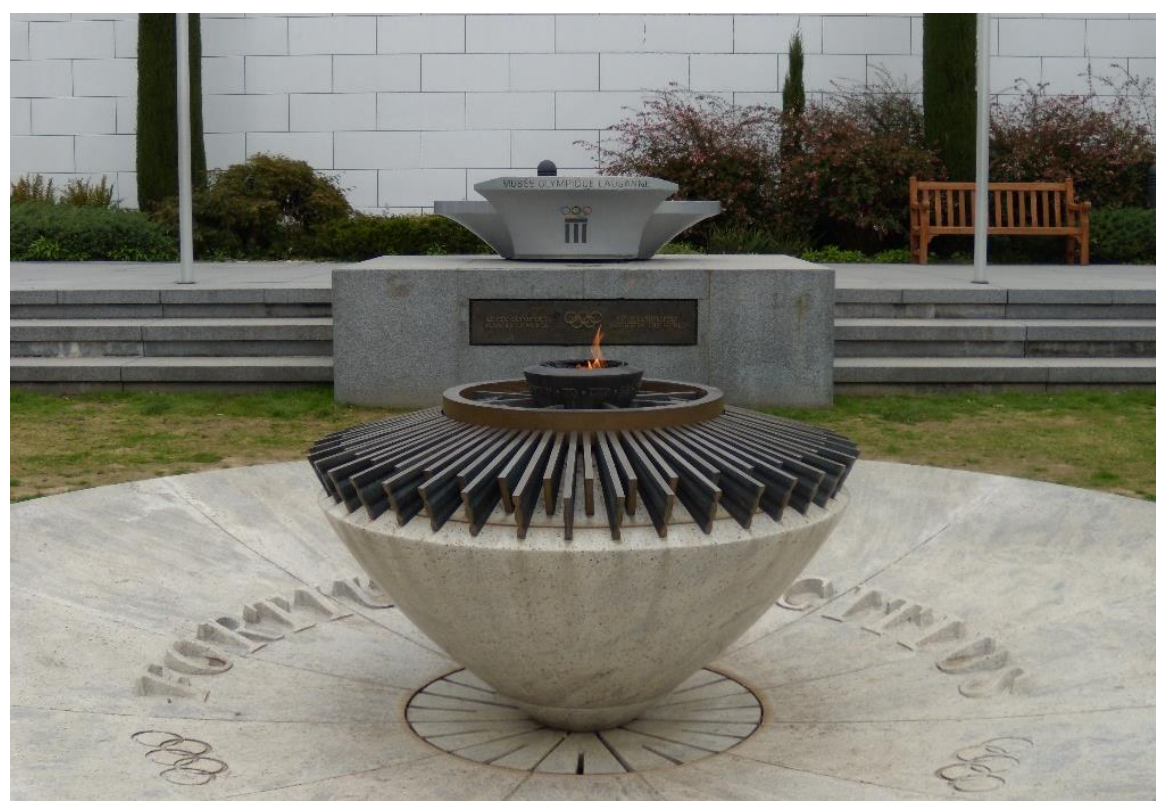

Foto \# 12 Monumento a la llama Olímpica. Museo Olímpico - André Ricard 1993. Foto archivo personal Carlos E. Villegas

\section{REFERENCIAS BIBLIOGRÁFICAS}

Barion, G. (2014). The Olympics tell the History through philatelic images. Tipografía Veneta.

COI. (2020). Carta Olímpica. In Vigente a partir del 17 de Julio de 2020 (pp. 103). Comité Olímpico Internacional.

Hirthler, G. (2016). The Idealist: The Story of Baron Pierre de Coubertin. Ringworks Press.

Kristy, D. (1995). Coubertin's Olympics: How the Games Began. Lerner Publications Company.

Miller, D. (2008). The official history of the Olympic Games and the IOC: Athens to Beijing, 1894-2008. Mainstream Publishing Company Limited.

Müller, N. (1994). One hundred years of Olympic Congresses 1894-1994. International Olympic Committee. 
Müller, N. (2000). Pierre de Coubertin 1863-1937: Olympism. Selected Writings. International Olympic Committee.

Müller, N., \& Schwank, W. (2008). Die olympische Devise" citius, altius, fortius" und ihr Urheber Henri Didon. ¿?

Ramos Vicent, M. P. (2012). Los Juegos Olímpicos en el arte. Editorial Planeta S.A.

Tarassouleas, A. (1988). Olympic Games in Athens: 1896, 1906. Vironas

Vatican-Philatelic-Society. (2000). Father Henri Didon Aerogramme. Crimando, Thomas. Vatican-Philatelic-Society. 